\title{
Temporal Parts and Complex Predicates
}

\author{
Thomas Sattig \\ Washington University in St. Louis \\ Proceedings of the Aristotelian Society 102 (2002): 279-286
}

Those who believe that ordinary things have temporal as well as spatial parts must give an account of the truth conditions of temporally modified predications of the form ' $a$ is $\mathrm{F}$ at $t$ ' in terms of temporal parts. I will argue that the friend of temporal parts is committed to an account of temporal predication that is incompatible with the classical principle of predicate abstraction.

\section{Temporal predication with temporal parts}

What is a temporal part of an object? To keep things simple, it will be helpful to draw the following analogy to space: just as places are spatial regions, times are temporal regions; and just as objects are located at, or occupy, spatial regions, objects are located at, or occupy, temporal regions. We can then say that just as a spatial part of an object occupies a sub-region of the spatial region occupied by the whole object, a temporal part of an object occupies a sub-region of the temporal region occupied by the whole object. $^{1}$

Assuming that there are objects and temporal parts of objects, which of these things are the particles and persons of our pre-philosophical ontology? The standard answer is that particles and persons are sums of temporal parts that are maximal under some 'unity relation'. There is, for instance, a unity relation for persons, and a person is a maximal sum of temporal parts, each of which stands in the unity relation for persons to all the others (and to itself). ${ }^{2}$ Moreover, not only ordinary objects but also their temporal

\footnotetext{
${ }^{1} 1$. Contemporary friends of temporal parts include Armstrong (1980), Heller (1990), Lewis (1983 + postscript B, 1986), Quine (1963), and Sider (2001).

${ }^{2}$ See Lewis (1983: 59).
} 
parts have various properties and relations. Temporal parts of persons, for instance, have a certain posture, height, and weight.

Friends of temporal parts describe ordinary objects in temporally insensitive terms; a person is said to have a temporal part simpliciter that has a certain weight simpliciter. But this is not how we speak about things on the street where a person is said to have a certain weight at a time. To explain the link, the friend of temporal parts must give truth conditions for temporal predications of the form ' $a$ is $\mathrm{F}$ at $t$ ' in terms of objects and their temporal parts. The obvious way to do this is to understand an object's being a certain way at a time in terms of the way its temporal part at this time is. More precisely, the truth conditions of monadic temporal predications take the following form:

$\left(\mathrm{TP}_{1}\right)$ Necessarily, ( $a$ is $\mathrm{F}$ ) at $t$ iff $a$ has a temporal part, $a_{t}$, that is located at $t \& a_{t}$ is $\mathrm{F}$.

The extension to the dyadic case is straightforward:

$\left(\mathrm{TP}_{2}\right)$ Necessarily, ( $a$ is $\mathrm{R}$ to $b$ ) at $t$ iff $a$ has a temporal part, $a_{t}$, that is located at $t \& b$ has a temporal part, $b_{t}$, that is located at $t \& a_{t}$ is $\mathrm{R}$ to $b_{t}$.

The temporal predications on the left-hand sides of $\left(\mathrm{TP}_{1}\right)$ and $\left(\mathrm{TP}_{2}\right)$ are regimented as containing a sentence-modifier 'at $t$ '. This is the standard construal of temporal operators in temporal predications. Alternatives will be considered shortly. Most friends of temporal parts have assumed a neutral attitude concerning the role of temporal operators. The problem to be discussed below provides a reason for being more concerned with matters of logical form. ${ }^{3}$

\section{The threat of predicate abstraction}

I will now show that the temporal-parts account of temporal predication stated as (TP1) and (TP2) is incompatible with the classical principle of predicate abstraction. The principle says that we can form a complex predicate from any open sentence. Accordingly, any sentence containing a singular term has an equivalent simple predication with this term in subject

\footnotetext{
${ }^{3}$. Since 'Charlie is happy at midnight' might have a different logical form from 'Charlie screams (hysterically) at midnight', the temporal-parts account of ' $a$ is $\mathrm{F}$ at $t$ ' should not be straightforwardly extended to ' $a$ Fs at $t$ '.
} 
position. For instance, 'Charlie is losing his mind' is equivalent to 'Charlie is such that he is losing his mind' which contains the complex predicate 'is such that he is losing his mind'. In general, for any sentence 'S(a)',

$(\mathrm{PA}) \square(\mathrm{S}(a) \equiv \lambda x[\mathrm{~S}(x)](a))$

Consider the identity-statement 'Charlie is identical to Charlie'. According to (PA), this statement is equivalent to 'Charlie is such that he is identical to Charlie'. Letting ' $c$ ' stand for Charlie, we have the following instance of (PA):

(1) $\square(c=c \equiv \lambda x[x=c](c))$

The left-hand side of (1) contains the simple two-place predicate of identity. The right-hand side of (1) contains the complex one-place predicate ' $\lambda x[x$ $=c]()^{\prime}$ ' which is obtained from the two-place predicate ' $=$ ', (or ' $\lambda x \lambda y[x$ $=y]($, )') by filling up one of its places by the constant ' $c$ '. The intuitive difference between the left-hand side and the right-hand side of (1) may be captured in the language of properties and relations: the left-hand side says that Charlie has the relation of identity to Charlie, whereas the right-hand side says that Charlie has the property of being identical to Charlie. The property of being identical to Charlie is often classified as a 'relational' property in the sense that Charlie's having it somehow 'consists in' having the relation of identity to himself. In the present context, such talk of properties and relations is merely a heuristic device. The argument to be given does not require taking a stand on whether there is a relational property corresponding to the predicate ' $\lambda x[x=c]$ ( )' or, more generally, whether there is a complex property corresponding to any of the complex predicates the predicate-abstraction principle allows us to form.

Now to temporal predication. Given that temporal operators function as sentence-modifiers, the predicate-abstraction principle (PA) has the following temporally modified variant:

$$
\square(\operatorname{At} t(\mathrm{~S}(a)) \equiv \operatorname{At} t(\lambda x[\mathrm{~S}(x)](a)))
$$

Consider the temporally modified identity-statement '(Charlie is identical to Charlie) at $t$ '. According to ( $\left.\mathrm{PA}^{*}\right)$, this statement is equivalent to '(Charlie is such that he is identical to Charlie) at $t$ ', just as 'Charlie is identical to Charlie' is equivalent to 'Charlie is such that he is identical to Charlie' by (PA). Or formally: 


$$
\square(\text { At } t(c=c) \equiv \operatorname{At} t(\lambda x[x=c](c)))
$$

Let us apply the temporal-parts account of temporal predication to the left-hand side and to the right-hand side of (2). The left-hand side contains a two-place predicate and therefore is an instance of $\left(\mathrm{TP}_{2}\right)$ :

(3) At $t(c=c)$ iff $c$ has a temporal part, $c_{t}$, that is located at $t \& c_{t}=$ $c_{t}$.

The right-hand side of (2) contains a one-place predicate and therefore is an instance of $\left(\mathrm{TP}_{1}\right)$ :

(4) At $t(\lambda x[x=c](c))$ iff $c$ has a temporal part, $c_{t}$, that is located at $t \&$ $\lambda x[x=c]\left(c_{t}\right)$.

Since it is clearly possible for Charlie to have a temporal part located at $t$ that is identical to itself, (3) is unproblematic. (4), on the other hand, creates serious trouble. Since Charlie is a temporally extended person, Charlie's instantaneous temporal part $c_{t}$ is a proper part of Charlie and hence distinct from him. It is therefore inconsistent to say that $c_{t}$ has the property of being identical to Charlie $-\lambda x[x=c]\left(c_{t}\right)$, because only Charlie has the property of being identical to Charlie. (I am assuming that ' $=$ ' is to be read as strict identity as opposed to, say, overlap.) This makes 'At $t(\lambda x[x=$ $c](c)$ )' trivially false; and so (2) is false. Hence, the temporal-parts account of temporal predication stated as $\left(\mathrm{TP}_{1}\right)$ and $\left(\mathrm{TP}_{2}\right)$ is incompatible with predicate abstraction.

The argument is not confined to the case of identity. Suppose, to give another example, that (Suzie is bored of Charlie) at $t$. According to (PA*), this statement is equivalent to '(Suzie is such that she is bored of Charlie) at $t$ '. Abbreviating the two-place predicate 'is bored of' as ' $\mathrm{B}($,$) ', we have$ the following instance of $\left(\mathrm{PA}^{*}\right)$ :

(5) $\square(\operatorname{At} t(\mathrm{~B}(s, c)) \equiv \operatorname{At} t(\lambda x[\mathrm{~B}(x, c)](s)))$

By $\left(\mathrm{TP}_{2}\right)$,

(6) At $t(\mathrm{~B}(s, c))$ iff $s$ has a temporal part, $s_{t}$, that is located at $t \& c$ has a temporal part, $c_{t}$, that is located at $t \& \mathrm{~B}\left(s_{t}, c_{t}\right)$.

By $\left(\mathrm{TP}_{1}\right)$, 
(7) At $t(\lambda x[\mathrm{~B}(x, c)](s))$ iff $s$ has a temporal part, $s_{t}$, that is located at $t$ $\& \lambda x[\mathrm{~B}(x, c)]\left(s_{t}\right)$.

Since it is consistent to suppose that it is true that $s_{t}$ has the relation of being bored-of to $c_{t}-\mathrm{B}\left(s_{t}, c_{t}\right)$ - but false that $s_{t}$ has the property of being bored of Charlie $-\lambda x[\mathrm{~B}(x, c)]\left(s_{t}\right),(5)$ is false.

A radical response to this result by the friend of temporal parts would be to reject the principle of predicate abstraction, (PA). It is an interesting question what costs would be involved in this move, but not a question to be pursued here. I will rather consider the more modest strategy of trying to reconcile the doctrine of temporal parts with (PA) and argue that it cannot be done.

\section{Temporal predication like spatial predication}

Temporal predication has a spatial analogue: just as something can be a certain way at a time, something can be a certain way at a place; just as a person can be happy at midnight, a road can be bumpy in the mountains. Since it is perfectly commonplace that ordinary objects have spatial parts, something's being a certain way at a place is naturally explained in terms of the way its spatial part in this place is. Assuming, in analogy with the temporal case, that spatial operators function as sentence-modifiers, we get the following account of the truth conditions of monadic and dyadic spatial predications:

$\left(\mathrm{SP}_{1}\right)$ Necessarily, $(a$ is $\mathrm{F})$ at $p$ iff $a$ has a spatial part, $a_{p}$, that is located at $p \& a_{p}$ is $\mathrm{F}$.

$\left(\mathrm{SP}_{2}\right)$ Necessarily, ( $a$ is $\mathrm{R}$ to $b$ ) at $p$ iff $a$ has a spatial part, $a_{p}$, that is located at $p \& b$ has a spatial part, $b_{p}$, that is located at $p \& a_{p}$ is $\mathrm{R}$ to $b_{p}$.

Now consider the statement '(road $r$ is parallel to road $\left.r^{*}\right)$ at $p$ '. By the spatially modified variant of the predicate-abstraction principle (the spatial analogue of $\left.\left(\mathrm{PA}^{*}\right)\right)$, this statement is equivalent to ' $r$ is such that it is parallel to $\left.r^{*}\right)$ at $p^{\prime}$. Letting ' $\mathrm{P}(,)^{\prime}$ ' abbreviate 'is parallel to', we get:

$$
\left(\text { At } p\left(\mathrm{P}\left(r, r^{*}\right)\right) \equiv \text { At } p\left(\lambda x\left[\mathrm{P}\left(x, r^{*}\right)\right](r)\right)\right)
$$

By $\left(\mathrm{SP}_{2}\right)$, the left-hand side implies that $r$ has a spatial part that is located at $p, r_{p}$, and $r^{*}$ has a spatial part that is located at $p, r^{*}{ }_{p}$, and $r_{p}$ has the relation of being parallel-to to $r^{*}{ }_{p}$. By $\left(\mathrm{SP}_{1}\right)$, the right-hand side of (8) 
implies that $r$ has a spatial part located at $p, r_{p}$, and $r_{p}$ has the property of being parallel to $r^{*}$. Since it is consistent to suppose that $r_{p}$ has the relation of being parallel-to to $r^{*}{ }_{p}$ without having the property of being parallel to $r^{*},(8)$ is false. Hence, the spatial-parts account of spatial predication stated as $\left(\mathrm{SP}_{1}\right)$ and $\left(\mathrm{SP}_{2}\right)$ is incompatible with $(\mathrm{PA})$. This result puts the threat of predicate abstraction in a different light, since it would be a violation of commonsense to deny the doctrine that ordinary objects have spatial parts. ${ }^{4}$

I can see two ways of removing the incompatibility, each of which concerns the logical form of temporal and spatial predications. The first suggestion is to construe the modifiers 'at $t$ ' and 'at $p$ ' as they occur on the left-hand sides of $\left(\mathrm{TP}_{1}\right),\left(\mathrm{TP}_{2}\right),\left(\mathrm{SP}_{1}\right)$, and $\left(\mathrm{SP}_{2}\right)$ not as sentence-modifiers, but rather as subject-modifiers, yielding in the monadic case ' $a$-at- $t$ is $\mathrm{F}$ ' and ' $a$-at- $p$ is F'. Given temporal and spatial parts, the singular terms ' $a$ at- $t$ ' and ' $a$-at- $p$ ' may be taken as abbreviations of the complex definite descriptions 'the temporal part of $a$ that is located at $t$ ' and 'the spatial part of $a$ that is located at p', respectively.

On this proposal, the unregimented temporal predication 'Charlie is identical to Charlie at $t$ ' becomes 'Charlie-at- $t$ is identical to Charlie-at$t$ '. According to (PA), this is equivalent to 'Charlie-at- $t$ is such that he is identical to Charlie-at- $t$ ':

$$
\square(c \text {-at- } t=c \text {-at- } t) \equiv(\lambda x[x=c \text {-at- } t](c \text {-at- } t)))
$$

The troublesome (2) follows from (PA) on the assumption that 'at $t$ ' in 'Charlie is identical to Charlie at $t$ ' is a sentence-modifier. If 'at $t$ ' is construed as a subject-modifier instead, then all (PA) yields is (9), which is unproblematic for the temporal-parts account of temporal predication. The other examples discussed receive the same treatment. Assuming the subjectmodifier reading, the temporal-parts account of temporal predication and the spatial-parts account of spatial predication therefore turn out to be compatible with (PA).

The second suggestion is to treat the left-hand sides of $\left(\mathrm{TP}_{1}\right)$ and $\left(\mathrm{TP}_{2}\right)$ as elliptical for their right-hand sides. Analogously for $\left(\mathrm{SP}_{1}\right)$ and $\left(\mathrm{SP}_{2}\right)$. Thus, 'Charlie is identical to Charlie at $t$ ' is short for 'Charlie has a temporal part that is located at $t, c_{t}$, such that $c_{t}$ is identical to $c_{t}$ '. Applying (PA) to ' $c_{t}=c_{t}$ ' yields a variant of $(9)$, and hence the ellipsis reading of temporal and spatial predications avoids the threat of predicate abstraction in the same straightforward way as the subject-modifier reading.

\footnotetext{
${ }^{4}$ Thanks to Jeremy Butterfield for pushing this point.
} 
The subject-modifier reading fails both for temporal predications and spatial predications. The problem with this reading is that it cannot handle cases in which the singular term in subject position is already modified. Consider the following spatial predication:

(10) The road that is bumpy in the mountains is smooth in the valley.

If the subject-modifier reading is applied to the singular term 'the road that is bumpy in the mountains', the latter designates the bumpy mountain-part of a road. Applying the subject-modifier reading to the whole predication is spatially to modify the singular term in subject position, 'the road that is bumpy in the mountains', to the effect that the modified term designates the valley-part of the road that is bumpy in the mountains. As we have just seen, however, 'the road that is bumpy in the mountains' designates the mountain-part of a road. Although a road can have a mountain-part and a valley-part, the mountain-part cannot have a valley-part. By contrast, the ellipsis reading has no difficulty dealing with this case:

(11) The road that [has a spatial part that] is bumpy [and that is located] in the mountains [has a spatial part that] is smooth [and that is located] in the valley.

Analogous considerations apply to such temporal predications as 'the person now sitting in the corner was standing at midnight'.

The ellipsis reading works for spatial predications but not for temporal predications. Linguists emphasise that the omitted parts of an ordinary elliptical sentence must be unambiguously specifiable by an ordinary speaker. For instance, in the sequence A: Where are you going? B: Home, the complete form of B's sentence is predictable from A's sentence ('I am going home'). Accordingly, an ordinary speaker, such as Suzie, must be able to specify the complete form of the sentence 'Charlie is happy at midnight' as 'Charlie has a temporal part that is happy and that is located at midnight'. But Suzie cannot do this. The concept of a temporal part of an object is a technical concept unavailable to the non-philosopher. Thus, ordinary speakers like Suzie lack this concept and hence lack the ability to specify the 'elided' parts of temporal predications. One might, in response, insist that there are elliptical sentences whose omitted parts are unspecifiable by ordinary speakers. But this would have the unacceptable consequence that ordinary language is systematically misleading. The situation is different in the spatial case. Ordinary speakers are perfectly able to specify the complete form of the sentence 'the road is bumpy in the mountains' as 'the road has a 
part that is bumpy and that is located in the mountains'. The reason is that the concept of a spatial part of an object is an ordinary, non-philosophical concept.

So the analogy breaks down: the ellipsis reading of spatial predications is a viable option, and hence the doctrine of spatial parts can avoid the threat of predicate abstraction, whereas the ellipsis reading of temporal predications is unacceptable, and hence the doctrine of temporal parts must face the threat of predicate abstraction. ${ }^{5}$

\section{References}

[1] Armstrong, D. M., 1980, 'Identity Through Time', in Time and Cause: Essays Presented to Richard Taylor, ed. P. van Inwagen, pp. 67-78. (Boston: Reidel).

[2] Heller, M., 1990, The Ontology of Physical Objects: Four Dimensional Hunks of Matter (Cambridge: Cambridge University Press).

[3] Lewis, D., 1983, 'Survival and Identity', in his Philosophical Papers, Vol. I. (Oxford: Oxford University Press), pp. 55-77.

[4] Lewis, D., 1986, On the Plurality of Worlds (Oxford: Blackwell).

[5] Quine, W.V.O., 1963, 'Identity, Ostension, and Hypostasis', in his From a Logical Point of View (New York: Harper and Row).

[6] Sider, T., 2001, Four-Dimensionalism: an Ontology of Persistence and Time (Oxford: Oxford University Press).

\footnotetext{
${ }^{5}$ I would like to thank Tim Williamson and my audience at the 2001 Joint Session for helpful comments.
} 